\title{
Fungal peritonitis in peritoneal dialysis patients: is previous antibiotic therapy an essential condition?
}

\author{
Nuno Guimarães Rosa, ${ }^{1}$ Sónia Silva, ${ }^{2}$ José António Lopes, ${ }^{2}$ Patrícia Branco, ${ }^{2}$ Edgar de Almeida, ${ }^{2}$ \\ Carlos Ribeiro, ${ }^{3}$ Fernando Abreu, ${ }^{2}$ José Barbas ${ }^{2}$ and M. Martins Prata ${ }^{2}$ \\ ${ }^{1}$ Department of Nephrology, Hospital Central do Funchal, Estrada dos Marmeleiros, Funchal, ${ }^{2}$ Department of Nephrology and Renal Transplantation and \\ ${ }^{3}$ Department of Clinical Pathology, Microbiology Laboratory, Hospital de Santa Maria, Lisboa, Portugal
}

\section{Summary}

\begin{abstract}
The aim of this study was to analyse the clinical and microbiological features of fungal peritonitis, in chronic peritoneal dialysis patients, focusing on non-traditional risk factors for this feared complication. From 2001 to 2004, five episodes of fungal peritonitis were diagnosed in five different patients, accounting for $4.5 \%$ of all peritonitis cases seen during this period. Candida spp. were the most frequent isolates. In all cases, peritoneal dialysis catheter removal and switching to haemodialysis were necessary. In these five cases of fungal peritonitis only one was preceded by antibiotic use, within the previous 3 months, the classical risk factor for fungal peritonitis. Identifying predisposing factors usually not taken into account, may lead to an early diagnosis and to a better understanding of fungal peritonitis pathogenesis.
\end{abstract}

Key words: fungal peritonitis, peritoneal dialysis, risk factors.

\section{Introduction}

Fungal peritonitis is a rare but serious complication of chronic peritoneal dialysis, responsible for $2.6-14.3 \%$ of peritonitis occurring in this set of patients. ${ }^{1-5}$ Fungal peritonitis has been traditionally associated with high morbidity and mortality, ${ }^{6,7}$ often leading to technique failure. Clinical presentation of fungal peritonitis is similar to bacterial peritonitis and Candida spp. is the most common isolate accounting for $70-89.3 \%$ of cases. $^{1,2,5,6}$ Previous antibiotic therapy is the most common risk factor associated to fungal peritonitis. ${ }^{1,2,5}$

The approach to fungal peritonitis has changed considerably in the last few years. In ISPD guidelines $2000,{ }^{8}$ fungal peritonitis was not considered a clear indication for catheter removal. More recent recommendations, ${ }^{7}$ however, have stressed the importance of early peritoneal catheter removal in association with

Correspondence: Dr Nuno Guimarães Rosa, Serviço de Nefrologia, Hospital Central do Funchal, Estrada dos Marmeleiros, 9054-535 Funchal, Portugal. Tel.: + 3512170 5730. Fax: + 351291724545

E-mail: guimaraesrosa@hotmail.com

Accepted for publication 25 August 2006 antifungal therapy, a procedure that has improved patients outcome. Another important aspect of fungal peritonitis management relates to determining antifungal sensitivities as imidazole resistant strains have been emerging. ${ }^{7}$ In order to highlight the importance of other fungal peritonitis risk factors, besides previous antibiotic therapy, we report five cases of fungal peritonitis that occurred in our Unit, four of which unrelated to this established risk factor.

\section{Patients and methods}

Between January 2001 and June 2004, a total of 95 patients with chronic kidney disease underwent peritoneal dialysis in our Unit. Fifty-seven (60\%) patients were on automated peritoneal dialysis and $38(40 \%)$ on continuous ambulatory peritoneal dialysis. Peritoneal dialysis access was achieved with a Tenckhoff peritoneal catheter inserted by laparoscopy.

We reviewed the clinical records of the five patients who had fungal peritonitis during this period. Fungal peritonitis diagnosis was made based on clinical and laboratorial features such as fever, abdominal pain, cloudy dialysate, leucocyte count in dialysate higher than $100 \mathrm{~mm}^{-3}$ with $>50 \%$ of neutrophils and a positive 
fungus culture in one or more occasions ${ }^{8}$ (ISPD Guidelines, 2000). Culture of peritoneal dialysate was performed by inoculation of $10 \mathrm{ml}$ of peritoneal effluent into a blood culture medium (BacT/Alert), that was incubated for 7 days at $37^{\circ} \mathrm{C}$ in an aerobic environment and by concentration of $40 \mathrm{ml}$ of peritoneal effluent followed by inoculation of the sediment on solid and liquid culture media (Blood agar, MacConkey agar, Sabouraud agar, Todd Hewitt broth and Beef infusion). These media were incubated for $48 \mathrm{~h}$ at $37^{\circ} \mathrm{C}$ in aerobiosis. For the isolation of fungi the incubation time of Sabouraud's agar was prolonged for a minimum of 7 days at room temperature. Identification of yeast was made using the Walkaway System with apropriate panels for automated identification (Microscan Rapid Yeast ID; Dade Behring Inc., West Sacramento, CA, USA). Antifungal sensitivity tests were not available in our institution.

\section{Results}

In the aforementioned period, a total of 105 episodes of bacterial peritonitis and $5(4.5 \%)$ episodes of fungal peritonitis, one per patient, were recorded. The incidence of fungal peritonitis was 0.03 fungal peritonitis/ patient/year. The isolates were Candida parapsilosis (two cases), Candida guilliermondii, Candida albicans and Rhodotorula rubra (one case each).

The patients were three men and two women with a mean age of 48 years (31-69). Mean time on peritoneal dialysis was 44.8 months (4-66). Chronic kidney disease aetiology was diabetic nephropathy in three patients, lupus nephritis in one patient and chronic glomerulonephritis in another.

All patients had abdominal pain and all, but one had a cloudy dialysate. Other clinical signs included vomiting, diarrhoea and peritoneal irritation. Three patients had ileus and two developed intra-abdominal abscess. Mean dialysate white cell count was $440 \pm 200{\text { cells } \mathrm{mm}^{-3}}^{-3}$ with $82 \% \pm 10.1 \%$ neutrophils. Only one patient had fungaemia (Candida albicans). Fungal peritonitis risk factors were identified in all patients (Table 1); only one patient had received antibiotherapy in the previous month for bacterial peritonitis.

All patients initiated antifungal therapy with an azole antifungal; oral fluconazole (three patients) or intra-peritoneal fluconazole (two patients) followed, in absence of clinical improvement, by oral itraconazole (one patient) and intravenous amphotericin B (all cases). Because of poor clinical response, all peritoneal catheters had to be removed within the first week of antifungal therapy. Peritoneal catheter tips were not cultured. In all cases, patients were switched to haemodialysis and none resumed peritoneal dialysis. One patient died 30 days after institution of antifungal therapy. She was a diabetic and suffered a fatal ischemic stroke.

\section{Discussion}

The prevalence of fungal peritonitis in our Unit was similar to that found in other series (2.6-14.3\%). Candida spp. contributed to $70-89.3 \%^{1,2,5,6}$ of fungal peritonitis episodes in different studies. In our series Candida spp. were responsible for $80 \%$ of fungal peritonitis episodes. Rhodotorula rubra, a rare but documented agent of fungal peritonitis, ${ }^{9}$ was isolated in one patient.

Mortality rate is variable but can be as high as $53.6 \%,{ }^{5}$ particularly, if catheter removal is delayed by $>24 \mathrm{~h}^{6}{ }^{6}$ The concern that prolonging peritoneal dialysis catheter in situ might be associated with higher mortality rate is reflected by the new ISPD Recommendations, ${ }^{7}$ in which fungal peritonitis is now a formal indication for immediate catheter removal. In all patients fungal peritonitis determined peritoneal dialysis dropout, which is in agreement with previous reports stating fungal peritonitis as an important cause of technique failure. ${ }^{6}$

Only one patient had previous antibiotic therapy, within the 3 months preceding the fungal peritonitis episode. This is traditionally considered as the classic risk factor for fungal peritonitis in peritoneal dialysis

Table 1 Risk factors for fungal peritonitis.

\begin{tabular}{|c|c|c|c|c|c|c|}
\hline $\begin{array}{l}\text { Risk factors } \\
\text { fungal peritonitis }\end{array}$ & Fungal isolate & $\begin{array}{l}\text { Prior } \\
\text { antibiotic use* }\end{array}$ & $\begin{array}{l}\text { Multiple } \\
\text { bacterial peritonitis }\end{array}$ & $\begin{array}{l}\text { Diabetes } \\
\text { mellitus }\end{array}$ & $\begin{array}{l}\text { Systemic lupus } \\
\text { erythematosus }\end{array}$ & Steroids \\
\hline Patient 1 & Candida parapsilosis & & $v$ & & & \\
\hline Patient 2 & Candida parapsilosis & & & & $\checkmark$ & $v$ \\
\hline Patient 3 & Candida guilliermondii & $\checkmark$ & $\checkmark$ & $\checkmark$ & & \\
\hline Patient 4 & Candida albicans & & & $\checkmark$ & & \\
\hline Patient 5 & Rhodotorula rubra & & & $\checkmark$ & & \\
\hline
\end{tabular}

*within the previous 3 months preceding the fungal peritonitis. 
patients. In fact, previous antibiotic therapy is the main predisposing clinical condition for fungal peritonitis, preceding $28.6-87.3 \%$ of fungal peritonitis episodes. ${ }^{1,2,5,10}$ In our patients, after reviewing literature, we were able to identify other less known fungal peritonitis risk factors: diabetes mellitus (three patients); systemic lupus erythematosus (one patient); use of steroids (one patient) and multiple episodes of bacterial peritonitis $>3$ months before fungal peritonitis episode (two patients). In two patients, we were able to identify more than one risk factor for fungal peritonitis. ${ }^{11,12}$ Diabetes mellitus was identified in $29-64.3 \%$ of the patients in different fungal peritonitis series. ${ }^{5,10,11}$ In our cohort, $60 \%$ of the patients were diabetic. In a retrospective study of 20 fungal peritonitis episodes, ${ }^{12}$ lupus patients had a higher incidence of fungal peritonitis and five in six patients with lupus were on steroids during fungal peritonitis episode. Patients with previous bacterial peritonitis developed fungal peritonitis more commonly ${ }^{10}$ and the frequent occurrence of the former was identified as a fungal peritonitis risk factor. ${ }^{12}$ Environmental sources of fungus were not studied because the fungal peritonitis were episodic and involved different fungus species.

\section{Conclusions}

We highlight that the absence of previous antibiotic therapy, in a peritoneal dialysis patient with a clinical suspicion of peritonitis, does not exclude fungus origin and should prompt the search for other less frequent risk factors such as diabetes mellitus, systemic lupus erithematosus, use of steroids and previous multiple episodes of bacterial peritonitis.

\section{References}

1 Goldie SJ, Kiernan-Tridle L, Torres C, Gorban-Brennan N, Dunne D, Kliger AS, Finkelstein FO. Fungal peritonitis in a large chronic peritoneal dialysis population: a report of 55 episodes. Am J Kidney Dis 1996; 28: 86-91.

2 Chan TM, Chan CY, Cheng SW, Lo WK, Lo CY, Cheng IK. Treatment of fungal peritonitis complicating continuous ambulatory peritoneal dialysis with oral fluconazole: a series of 21 patients. Nephrol Dial Transplant 1994; 9: $539-42$.

3 Zelenitsky S, Barns L, Findlay I, Alfa M, Ariano R, Fine A, Harding G. Analysis of microbiological trends in peritoneal dialysis-related peritonitis from 1991 to 1998. Am J Kidney Dis 2000; 36: 1009-13.

4 Manzano-Gayosso P, Hernandez-Hernandez F, MendezTovar LJ, Gonzalez-Monroy J, Lopez-Martinez R. Fungal peritonitis in 15 patients on continuous ambulatory peritoneal dialysis (CAPD). Mycoses 2003; 46: 425-9.

5 Prasad KN, Prasad N, Gupta A, Sharma RK, Verma AK, Ayyagari A. Fungal peritonitis in patients on continuous ambulatory peritoneal dialysis: a single centre Indian experience. J Infect 2004; 48: 96-101.

6 Wang AY, Yu AW, Li PK, Lam PK, Leung CB, Lai KN, Lui SF. Factors predicting outcome of fungal peritonitis in peritoneal dialysis: analysis of a 9-year experience of fungal peritonitis in a single center. Am J Kidney Dis 2000; 36: 1183-92.

7 Piraino B, Bailie GR, Bernardini J, Boeschoten E, Gupta A, Holmes C, Kuijper EJ, Tao Li PK, Lye WC, Mujais S, Paterson DL, Fontan MP, Ramos A, Schaefer F, Uttley L. ISPD guidelines/recommendations. Peritoneal dialysisrelated infections: 2005 update. Perit Dial Int 2005; 25: 107-131.

8 Keane W, Bailie G, Boeschoten E, Gokal R, Golper T, Holmes C, Kawaguchi Y, Piraino B, Riella M, Vas S. ISPD guidelines/recommendations. Adult peritoneal dialysisrelated peritonitis treatment recommendations: 2000 update. Perit Dial Int 2000; 20: 396-411.

9 Eisenberg ES, Alpert BE, Weiss RA, Mittman N, Soeiro R. Rhodotorula rubra peritonitis in patients undergoing continuous ambulatory peritoneal dialysis. Am J Med 1983; 75: 349-52.

10 Wong PN, Mak SK, Lo KY, Tong GM, Wong AK. A retrospective study of seven cases of Candida parapsilosis peritonitis in CAPD patients: the therapeutic implications. Perit Dial Int 2000; 20: 76-9.

11 Kleinpeter MA, Butt AA. Non Candida albicans fungal peritonitis in continuous ambulatory peritoneal dialysis patients. Adv Perit Dial 2001; 17: 176-9.

12 Huang JW, Hung KY, Wu KD, Peng YS, Tsai TJ, Hsieh BS. Clinical features of and risk factors for fungal peritonitis in peritoneal dialysis patients. J Formos Med Assoc 2000; 99: 544-8. 